\section{Initial Postcritical Analysis of Asymmetric Bifurcation in Frames}

By Zdenčk P. Bažant,' Fellow, ASCE, and Luigi Cedolin, ${ }^{2}$ Member, ASCE

ABSTRACr: Asymmetry of the shape, supports, or loading of frames causes the initial post critical behavior to exhibit asymmetric bifurcation. Its source is the second-order axial shortening of columns due to lateral deflection, which induces asymmetric shear forces and bending moments transmitted into the column from
the adjacent beams. As an alternative to Koiter. Roorda, and Chilver's solutions the adjacent beams. As an alternative to Koiter, Roorda, and Chilver's solutions the paper presents a simpler and more general method of analysis that uses the stiffness matrices and stability functions from linear stability theory of frames. The usual linear matrix equilibrium equations are enhanced by additional terms that are quadratic in joint rotations, with coefficients that depend on the derivatives of the As an example, Koiter and Roorda's L-frame is analyzed and the results are shown to agree with the previous solutions as well as Roorda's experiments. It is concluded that the decrease of the maximum toad due to asymmetric bifurcation is not insignificant and should be taken into account in design.

\section{INTRODUCTION}

Due to asymmetry of shape, supports, or loading, the initial postcritical buckling behavior of many frames exhibits asymmetric bifurcation. As shown by Koiter (1945) and others, this behavior causes strong sensitivity to imperfections. As confirmed by the present paper, this sensitivity can cause the maximum load of an imperfect frame to be significantly lower than that of the perfect frame.

The general analysis of postcritical behavior is rather complicated, since it necessitates a geometrically nonlinear theory of bending. The initial postcritical behavior, the knowledge of which is sufficient for most practical purposes, can be obtained by power-series expansions of the nonlinear functions involved, particularly the curvature-deflection relation and the equilibrium conditions for the deflected structure. The postcritical analysis of asymmetric bifurcation based on power-series expansions has been demonstrated by Koiter (1967) by means of an example of a simple L-shaped frame. Roorda $(1965 a, b)$ conducted model experiments, which confirmed Koiter's calculations, particularly the reduction of the maximum load as a function of the initial imperfection in the form of load eccentricity. Koiter's analysis was based on the potential energy expression for the structure, which provides complete information for both equilibrium states and their stability. Roorda and Chilver (1970) showed a somewhat simpler method of analysis, which was based solcly on equilibrium equations and employed the perturbation method with power-series expansions. Although this analysis does not deal 'Prof. of Civ. Engrg., Northwestern Univ., Evanston, IL 60208.

'Prof., Dept. of Struct. Engrg., Politecnico DiMilano, Piazza Leonardo da Vinci, 32, 20133, Milan, Italy, and visiting Scholar, Northwestern Univ., Evanston, IL. Note. Discussion open until April 1, 1990. To extend the closing date one month, a written request must be filed with the ASCE Manager of Journals. The manuscrip for this paper was submitted for review and possible publication on January 15, 1989. This paper is part of the Journal of Structural Engineering $\mathrm{Vol} 115$, November 1989. ASCE, ISSN 0733-9445/89/0011-2845/\$1.00 + \$.15 Ner page. Paper No. 24064. with stability of equilibrium states, it yields all the information needed for practical purposes, and was shown by Roorda and Chilver to agree with Koiter's previous solution.

The previous solutions, however, still have certain shortcomings: (1) They rely solely on mathematical manipulations and do not provide much insight into the physical source and mechanism of the imperfection sensitivity; and (2) they are relatively complicated, because the nonlinear differential equations are integrated directly, without exploiting the existing powerful matrix method for frames.

The purpose of the present paper is to show a relatively simple method, which represents an adaptation of the stiffness matrix method for linear buckling analysis of frames. This new method directly introduces into the matrix equilibrium relations the physical source of bifurcation asymmetry, which consists in postcritical change in the axial force of a member caused: (1) By the asymmetric incremental shear forces transmitted from the adjoining members meeting at an angle; and (2) by the fact that the second-order axial shortening of a member due to joint rotation to one side meets less resistance than that due to joint rotation to the opposite side.

Another simplified method for nonlinear postcritical analysis of frames has recently been presented by Kounadis (1985) and illustrated by the example of the Koiter-Roorda L-frame. This method relies on direct integration of the differential equations for the deflection curves of members and is approximate since it achieves simplification by relaxing in a certain intuitive manner the compatibility conditions at the joints. By contrast the present solution does not require integration of differential equations, as it uses the stiffness matrices with stability functions, is asymptotically exact, and gives information on the magnitude of the contributions to imperfection sensitivity from various mechanisms.

It must, of course, be recognized that the complete postcritical behavior of perfect frames as well as the complete nonlinear response of imperfect frames can be calculated by the existing geometrically nonlinear finite element programs for beam structures, using the technique of step-by-step loading with iterations (e.g., Bažant and EINimeiri 1973). However, such programs are unnecessarily complicated when only the initial postcritical behavior is of interest, as is the case for most design purposes. They do not provide the designer the same insight as does a simpler, more direct solution, such as the one to be attempted here.

The analysis that follows assumes perfectly elastic behavior and applicability of the bending theory in which the bending moment is proportional to the curvature of the beam.

\section{L-Frame of Kotrer and RoOrda}

As is well known (e.g., Horne and Merchant 1965), the initial incremental stiffness matrix of a beam of uniform bending stiffness $E I$, subjected to axial force $P$, may be expressed as

$\left\{\begin{array}{c}M_{a} \\ M_{b} \\ V\end{array}\right\}=\frac{E I}{l}\left[\begin{array}{ccc}s & s c & \bar{s} / l \\ s c & s & \bar{s} / l \\ \bar{s} / l & \bar{s} / l & s^{*} / l^{2}\end{array}\right]\left\{\begin{array}{c}\theta_{a} \\ \theta_{b} \\ \Delta\end{array}\right\}$

in which $l=$ beam length; $\theta_{a}, \theta_{b}, \Delta=$ end rotation and relative transverse 


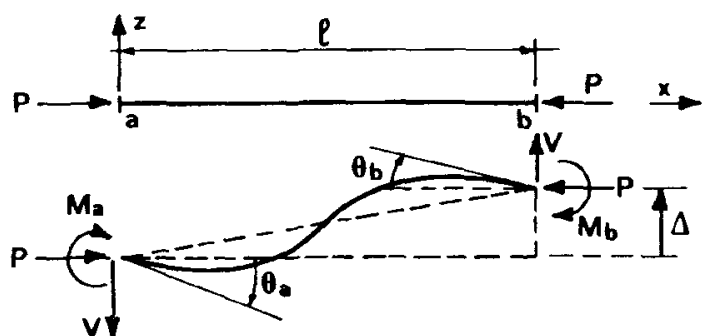

FIG. 1. Beam Element Subjected to End Forces and Moments

displacement of beam ends; $M_{a}, M_{b}$, and $V=$ associated end moments and shear force (Fig. 1); and $s, c, \bar{s}$, and $s^{*}=$ stability functions defined as follows:

for $P>0$ (compression): $\quad s=\frac{\gamma(\sin \gamma-\gamma \cos \gamma)}{2-2 \cos \gamma-\gamma \sin \gamma}$,

$c=\frac{\gamma-\sin \gamma}{\sin \gamma-\gamma \cos \gamma}$

for $P=0: \quad s=4, \quad c=\frac{1}{2}$

for $P<0$ (tension): $\quad s=\frac{\gamma(\gamma \cosh \gamma-\sinh \gamma)}{2-2 \cosh \gamma+\gamma \sinh \gamma}$,

$c=\frac{\sinh \gamma-\gamma}{\gamma \cosh \gamma-\sinh \gamma}$

with $\bar{s}=s(1+c), s^{*}=2 \bar{s}-\pi^{2} P / P_{E}, \gamma=\pi \sqrt{\rho}, \rho=P / P_{E}$, and $P_{E}=$ $E I \pi^{2} / l^{2}=$ Euler load

As an example, consider the L-frame shown in Fig. 2. In their famous, by now classic, papers Koiter (1967) used this example to illustrate asymmetric bifurcation and Roorda (1965a,b) confirmed Koiter's thcoretical predictions by experiment. The bars have equal uniform bending rigidities $E I$, and the ratio of their lengths is $\beta$. The vertical load $P$ is applied at the corner with a small eccentricity $e$. The deformation of the frame is characterized by rotations $\theta, \theta_{2}$, and $\theta_{3}$ [Fig. $\left.2(a, b)\right]$. The column and beam are assumed to be so slender that their furst-order axial shortenings due to axial forces are negligible.

The deflections $w$ produce second-order axial shortenings of the column and the beam. They are second-order small in terms of $w$ or $\theta$ and cause joint displacements $u^{(2)}$ downward and $v^{(2)}$ to the right [Fig. 2(a,b)].

Due to these displacements, buckling of the column to the right [Fig. 2(b)] produces an incremental shear force $V^{b}$ in the beam, which tends to make the axial compression force $P^{c}$ in the column larger than the applied load $P$. On the other hand, for buckling to the left, the shear force $V^{b}$ is of opposite sign and tends to make $P^{c}$ less than $P$ [Fig. 2(a)]. This favors buckling to the right. Secondly, buckling of the column to the right [Fig. 2(b)] pro- a)

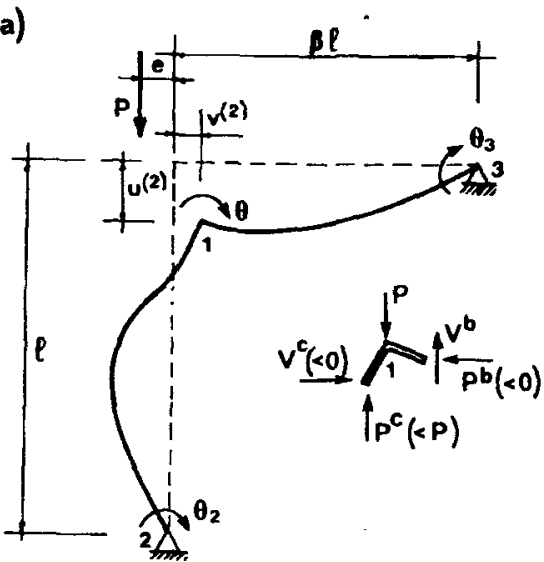

b)

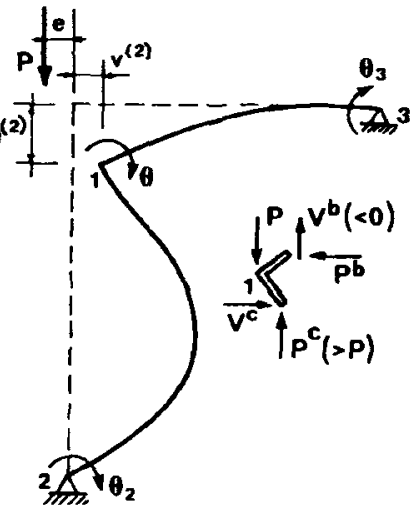

c)

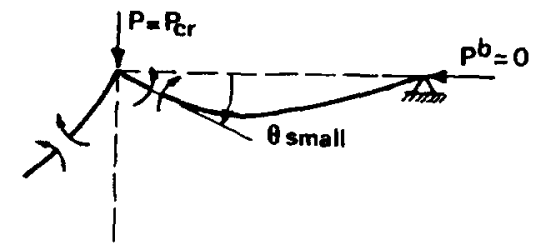

FIG. 2. Kolter-Roorda L-Frame: (a) Buckling to Left; (b) Buckling to Right; (c) Neutral Equilibrium at $P=P_{c r}$ for Perfect Frame (and Small $\theta$ )

duces a smaller curvature of the beam than buckling to the left [Fig. 2(a)], since for both cases the joint moves down and to the right. This, too, favors column buckling to the right [Fig. 2(b)]. Thirdly, buckling of the column to the right [Fig. $2(b)$ ] produces an incremental shear force in the column, which translates into a compressive force in the beam, lowering its stiffness, while buckling to the left causes a tensile force in the beam, increasing its stiffness. This again favors buckling to the right.

So we see that the response does not exhibit the same symmetry that is found for centrically loaded perfect columns, which are equally likely to buckle left or right. This asymmetry is manifested in the load-deflection diagram of an imperfect column and has an important consequence: it causes the maximum load to become less than $\boldsymbol{P}_{c r}$. The writer analysis will demonstrate it.

Taking $\boldsymbol{u}^{(2)}$ and $v^{(2)}$ into account but still considering small deflections, one obtains from Eq. 1 the following moment equilibrium condition of the joint (Fig. 2):

$\frac{E I}{l}\left(s_{c} \theta+s_{c} c_{c} \theta_{2}-\bar{s}_{c} \frac{v^{(2)}}{l}\right)+\frac{E I}{\beta l}\left(s_{b} \theta+s_{b} c_{b} \theta_{3}+\bar{s}_{b} \frac{u^{(2)}}{\beta l}\right)$

where $s_{c}, c_{c}$, and $\bar{s}_{c}=$ functions $s, c$, and $\bar{s}$ for the column, depending on $\rho^{c}=P^{c} / P_{E}^{c}\left(P^{c}=\right.$ axial compressive force in the column); and $P_{E}^{c}=$ Euler load of the column; $s, c_{b}$, and $\bar{s}_{b}=$ functions $s, c$, and $\bar{s}$ for the beam, 
depending on $\rho^{b}=P^{b} / P_{E}^{b}\left(P^{b}=\right.$ axial load of the beam and $P_{E}^{b}=$ Euler load of the beam).

The moment equilibrium conditions at hinges 2 and 3 read $s_{c} \theta_{2}+s_{c} c_{c} \theta$ $-\bar{s}_{c} v^{(2)} / l=0$ and $s_{b} \theta_{3}+s_{b} c_{b} \theta+\bar{s}_{b} u^{(2)} / \beta l=0$. Expressing $\theta_{2}$ and $\theta_{3}$ from these conditions and substituting them into Eq. 3, one obtains

$\left[s_{c}\left(1-c_{c}^{2}\right)+\frac{s_{b}}{\beta}\left(1-c_{b}^{2}\right)\right] \theta+s_{c}\left(c_{c}^{2}-1\right) \frac{v^{(2)}}{l}+\frac{s_{b}}{\beta}\left(1-c_{b}^{2}\right) \frac{u^{(2)}}{\beta l}=-\frac{P e l}{E I} \ldots$

The horizontal and vertical equilibrium conditions for the joint (Fig. 2) can be written as

$P^{b}=V^{c}=\frac{E I}{l^{2}} s_{c}\left(1-c_{c}^{2}\right) \theta$

and

$P^{c}=P-V^{b}, \quad V^{b}=\frac{E I}{(\beta l)^{2}} s_{b}\left(1-c_{b}^{2}\right) \theta$

where $V^{c}$ and $V^{b}=$ the shear forces in the column and the beam (Fig. 2). In the calculation of $V^{c}$ and $V^{b}$, higher-order terms have been omitted since the validity of the present theory is limited to small displacements.

Let us now determine $u^{(2)}$ and $v^{(2)}$. Small deflections of the members of the frame may be expressed as $w=\theta f_{c}(x)$ for the column and $w=\theta f_{b}(x)$ for the beam, where $x$ is the axial coordinate of the column or beam measured from the joint; $f_{c}(x)=A_{c} \sin k x+B_{c} \cos k x+C_{c} x+D_{c} ; k=\gamma / l$; and $f_{b}(x)=A_{b} x^{3}+B_{b} x^{2}+C_{b} x+D_{b}$. The expression adopted for $f_{b}(x)$ is a cubic polynomial, which is exact for $P^{b}=0$, and is always a sufficient approximation since $P^{b}$ (unlike $P^{c}$ ) is small (this will be confirmed later). The constants $A_{1}, B_{c}, \ldots, D_{b}$ are determined from the conditions $w=0$ and $w^{\prime}=1$ for $x=0$; and $w=0, w^{\prime \prime}=0$ for $x=l$ or $x=\beta l$, where the primes denote derivatives (here with respect to $x$ ). This yields $A_{c}=l /(\sin \gamma-\gamma$ $\cos \gamma) ; C_{c}=-\left(A_{c} / l\right) \sin \gamma ; B_{c}=D_{c}=0 ; A_{b}=-C_{b} / l^{2} ; C_{b}=1 / 2 ; B_{b}=$ $D_{b}=0$. Since the beam axis may be considered to be inextensible during buckling (as the axial force is negligible), we have $u^{(2)}$ or $v^{(2)}=\int(1-\cos$ $\left.w^{\prime}\right) d x=\int\left[1-\left(1-w^{\prime 2} / 2\right)\right] d x=\int 1 / 2 w^{\prime 2} d x$. Consequently, for small $\theta$

$u^{(2)}=k_{c} \theta^{2}, \quad k_{c}=\int_{0}^{\prime} \frac{1}{2}\left[f_{c}^{\prime}(x)\right]^{2} d x$.

$v^{(2)}=k_{b} \theta^{2}, \quad k_{b}=\int_{0}^{\beta l} \frac{1}{2}\left[f_{b}^{\prime}(x)\right]^{2} d x$

where coefficients $k_{b}$ and $k_{c}$ are positive. Their values are found to be $k_{b}=$ $0.1 \beta l$ and $k_{c}=A_{c}^{2}\left(2 \gamma^{2}+\gamma \sin 2 \gamma-4 \sin ^{2} \gamma\right) / 8 l$. Substituting Eqs. $7 a, b$ into Eq. 4 , one gets

$\left[s_{c}\left(1-c_{c}^{2}\right)+\frac{s_{b}}{\beta}\left(1-c_{b}^{2}\right)\right] \theta+\left[s_{c}\left(c_{c}^{2}-1\right) \frac{k_{b}}{l}+s_{b}\left(1-c_{b}^{2}\right) \frac{k_{c}}{\beta^{2} l}\right] \theta^{2}$

$=-\frac{P e l}{E I}$. a)

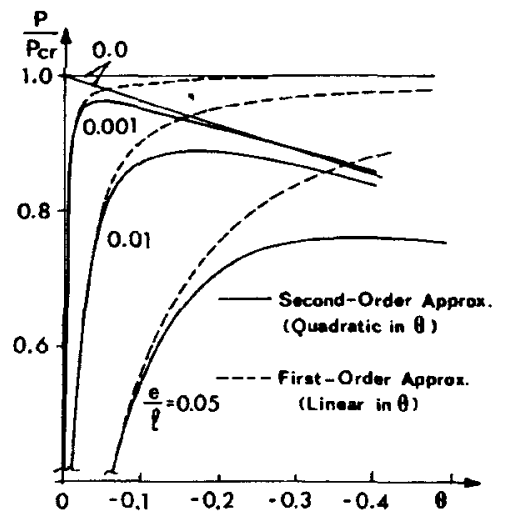

b)

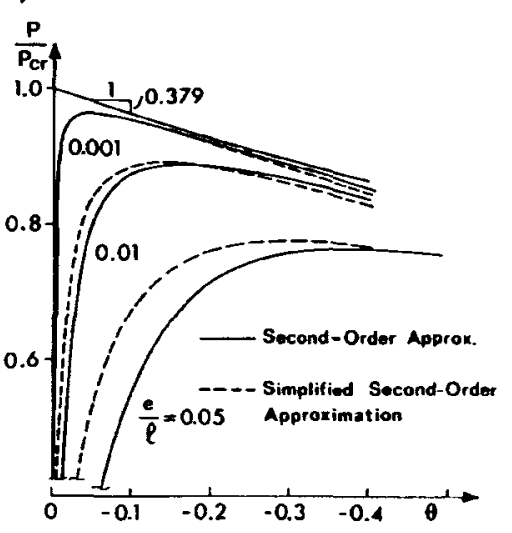

c)
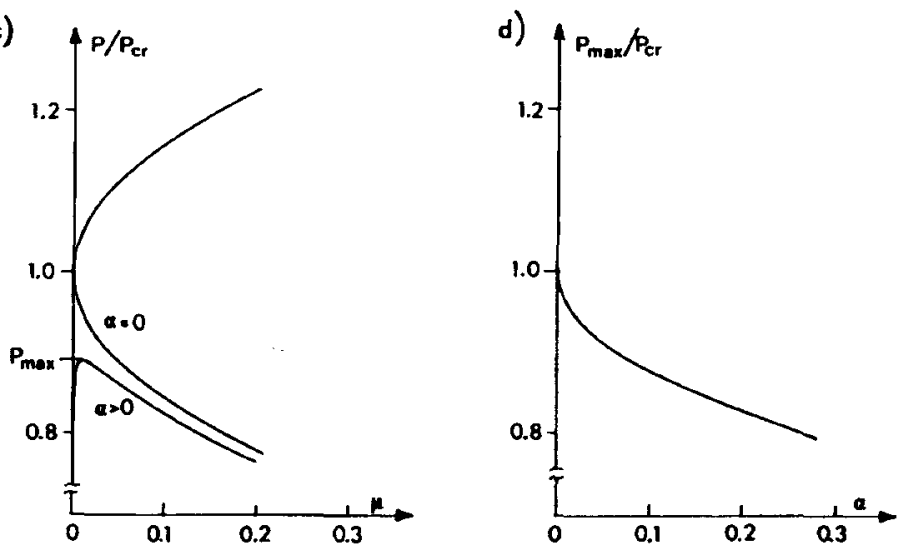

FG. 3. (a,b) Curves of Laad versus Rotation; (c) Curve for Load versus LoadPoint Displacement; (d) Imperfection Sensitivity Diagram

It is clear that if we would correct the deflection shapes $f_{c}(x)$ and $f_{b}(x)$ by taking $u^{(2)}, v^{(2)}$ into account, we would introduce into Eq. 8 only terms, whose order is higher than $\theta^{2}$ but would not change the terms with $\theta$ and $\theta^{2}$. Also note that since $P^{b}=0$ for $\theta 0$, the consideration of $P^{b} \neq 0$ in the expression of $f_{b}(x)$ would add a term proportional to $\theta$ in the expression of $k_{b}$ (Eq. $7 b$ ), which would translate into a higher-order term in Eq. 8.

Eqs. 5,6 , and 8 represent a system of five equations relating $\theta, P, P^{b}$, $P^{c}, V^{b}$, and $V^{c}$. If $P^{b}, P^{c}, V^{b}$, and $V^{c}$ are eliminated, one gets the relation of $P$ to $\theta$. For a convenient calculation of the curve $P(\theta)$, one may choose a series of closely spaced increasing values of $P^{c}$. For each $P^{c}$, one evaluates $s_{c}$ and $c_{c}$. Then, using as an approximation the previous rather than current value of $\theta$ in Eq. 5, one solves from it $P^{b}$ and then evaluates $s_{b}$ and $c_{b}$, upon which one solves two values from Eq. 8 (a quadratic equation), giving different portions of the $P(\theta)$ curve. Accuracy could be improved by iterating 
the procedure with the latest value of $\theta$ used in Eq. 5, but this is not necessary if the chosen $P^{c}$ values are very closely spaced.

The curves $P(\theta)$ are plotted in Fig. 3(a) for various values of relative eccentricity $e / l$. As expected, for $e>0$ the rotation $\theta$ is negative. A crucial fact to note is that, for $e>0$, the curve $P(\theta)$ has a maximum, while the curves that are obtained upon neglecting $u^{(2)}$ and $v^{(2)}$ [dashed line in Fig. 3(a)] do not. As a limit case for $e=0$, we obtain the initial post-critical response of the perfect system.

\section{Second-Ordea Solution of L-FaAme}

As has already been shown, the foregoing solution has only second-order (quadratic) accuracy in $\theta$. Therefore, any simplifications that preserve the second-order accuracy are admissible and cause no error. To this end, we expand all the variables in Eq. 8 into a power series with respect to $\theta$ about the critical state $(e=0)$. Then we discard all the terms of powers higher than $\theta^{2}$, i.e., solve Eq. 8 with second-order accuracy in $\theta$. Since we are expanding all the variable coefficients about the critical state, we evaluate the expansion coefficients for the critical state (onset of buckling, $\theta=0$ ). At that state, $u^{(2)}=v^{(2)}=0 ; P=P_{c r}=P^{c} ;$ and $V^{b}$ and $V^{c}=0$. Using power-series expansions for functions $s$ and $c$ (see Dean and Ugarte 1968), the second-order approximations for $s_{b}$ and $c_{b}$ near the critical state are

$s_{b}=4-\frac{2 \pi^{2}}{15} \frac{P^{b}}{P_{E}^{b}}, \quad c_{b}=\frac{1}{2}+\frac{3}{8} \frac{\pi^{2}}{15} \frac{P^{b}}{P_{E}^{b}}$

because $P^{b}=0$ at the critical state.

The approximation for $P^{b}$ as a function of $\theta$ can be obtained from Eq. 5 , but it can be more directly reasoned from the existence of neutral equilibrium at the onset of buckling [Fig. 2(c)]. Therefore, the moment acting on the column must be equal to the moment acting on the beam, which equals $3 E I /$ $\beta l$ because initially $P^{b}=0$. Then, using Eq. 5 , one may calculate the shear force in the column:

$P_{b}=V^{c}=-\frac{3 E I}{\beta l^{2}} \theta, \quad \rho^{b}=\frac{P^{b}}{P_{E}^{b}}=-\frac{3 \beta}{\pi^{2}} \theta$

From this it appears that $P^{b}>0$ (compression) if $\theta<0$. So we conclude that the change of beam stiffness also contributes to the initial downward slope for $\theta<0$.

Substituting $p^{b}$ from Eq. 10 into Eq. 9 and then $s_{b}$ and $c_{b}$ into Eq. 8, and neglecting terms that contain powers higher than $\theta^{2}$, we obtain

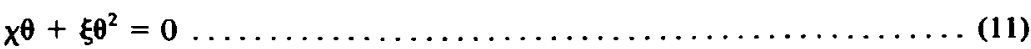

where $\chi=s_{c}\left(1-c_{c}^{2}\right)+3 / \beta$ and $\xi=s_{c}\left(c_{c}^{2}-1\right) k_{b} / l+3 k_{c} / \beta^{2} l+3 \beta / 5$. Coefficient $\chi$ depends on $\rho^{c}=P^{c} / P_{E}^{c}$. For $P$ close to $P_{c r}$

$\chi=\chi_{c r}+\left(P^{c}-P_{c r}\right) \chi^{\prime}, \quad$ with $\chi^{\prime}=\frac{d \chi}{d P^{c}}$

Now $\chi=0$ for $P=P_{c r}$, and so $\chi_{c r}=0$. (The reason is that at the onset of buckling all the second-order terms in Eq. 8 or Eq. 11 disappear.) This condition also gives the critical load of the perfect frame: $P_{c r}=\rho_{c r}^{c} P_{E}^{c}$, in which $\rho_{c r}=1.407$ forr $\beta=1$. The value of $\chi^{\prime}=(d \chi / d \rho) / P_{E}^{c}$ is evaluated at $p^{c}=P_{c r} / P_{E}^{c}$, and it may be noted that $\chi^{\prime}<0$. In view of Eq. 6 we have $\chi=\chi^{\prime}\left(P-V^{b}-P_{c r}\right)$. Taking into account Eq. 9 and neglecting third-order terms in $\theta, E .11$ becomes

$P=P_{c r}(1+a \theta)$

in which

$$
\begin{aligned}
& a=a_{1}+a_{2}+a_{3}, \quad a_{1}=\frac{3 E I}{(\beta)^{2} P_{c r}}, \\
& a_{2}=\frac{3 \beta}{5\left(-\chi^{\prime}\right) P_{c r}}, \quad a_{3}=\frac{\xi_{c r}}{\left(-\chi^{\prime}\right) P_{c r}} .
\end{aligned}
$$

Note that in writing Eq. 13 we neglected the increment $\Delta \xi=\left(P^{c}-P_{c r}\right) \xi^{\prime}$ because $\left(P^{c}-P_{c r}\right)$ is a small quantity that would be multiplied by $\theta^{2}$ when substituted in $\mathrm{Eq}$. 11 , and thus it would yield a third-order small term. In Fig. $3(b)$, Eq. 13 is represented by a straight line of slope $a$, which is very close to the solution previously obtained from the full equation system for $e=0$, and is asymptotically (i.e., for $\theta \rightarrow 0$ ) the same.

There are three terms that contribute to the slope $d P / d \theta$ : (1) The stiffness change of the column caused by its axial force change due to the vertical shear force transmitted to it from the beam (term $\left.a_{1}\right) ;(2)$ the stiffness change of the beam caused by its axial force change due to the horizontal shear force transmitted to it from the column (term $a_{2}$ ); and (3) the displacement of the corner due to axial shortenings of the beam and column caused by their deflections (term $a_{3}$ ). Note that in a symmetric frame the shear forces represented by $a_{1}$ as well as $a_{2}$ would be cancelled by the shear force from the opposite member, and term $a_{3}$ would vanish also if the joint displacement were precluded by symmetry.

The diagram of load versus load-point displacement $u\left(u=u^{(2)}\right)$ at $e=0$

(Fig. 2) is obtained, according to Eq. 7a, by substituting $\theta= \pm \sqrt{u / k_{c}}$ (with $k_{c}$ evaluated for $\left.P^{c}=P_{c r}\right)$, i.e.

$P=P_{c r}\left(1 \pm \frac{a}{\sqrt{k}_{c}} \sqrt{u}\right)$

Eq. 15 is plotted in Fig. 3(c).

For small values of $e$ and $\theta$, and for values of $P^{c}$ not too different from $P_{\mathrm{cr}}$, introduction of the approximations in Eqs. 9, 10, and 12 into Eq. 8 and elimination of higher-order terms in $\theta$ provides

$$
P=P_{c r} \frac{1+a \theta}{1-\left(\frac{\alpha}{\theta}\right)}, \quad \text { with } \alpha=\frac{l e}{E I\left(-\chi^{\prime}\right)}=\frac{\pi^{2}}{\frac{-d \chi}{d \rho^{c}}} \frac{e}{l}
$$

where $\alpha$ represents a nondimensional imperfection. Fig. 3(b) compares the solution of the full second-order nonlinear system of Eqs. 5-8 with the simplified second-order solution (Eq. 16). We may observe that for very small imperfections $(e / l=0.0001, e / l=0.001)$ these two second-order solutions 
agree closely. For stronger imperfections this is not so, mainly because $P$ is no longer close to $P_{c r}$.

\section{IMPERFECTION SENSITIVITY}

In Eq. 16 we may now introduce the expansion $1 /(1-\alpha / \theta)=1+(\alpha /$ $\theta)+(\alpha / \theta)^{2}+\ldots$ and assume that $\theta>>$ at maximum load. Then, neglecting in the resulting expression all higher-than-linear terms in $\theta$, we get

$\frac{P}{P_{c r}} \simeq 1+\frac{\alpha}{\theta}+a(\theta+\alpha)$

Setting $d P / d \theta=0$, the value of $\theta$ at maximum $P$ is obtained as $\theta_{m}=$ $-\sqrt{\alpha / a}$ (only the negative root is of interest because we know the column deflects to the right). Substituting this into Eq. 17 , we obtain $P_{\max } / P_{c r}=1$ $-2 \sqrt{a \alpha}-a \alpha$, and for small imperfections $\alpha$ we have

$P_{\max }=P_{c r}(1-2 \sqrt{a \alpha})$.

Eq. 18 is plotted in Fig. 3(d).

It is important to note that after reaching the critical state the load declines with increasing deflections; i.e., the structure exhibits softening. The diagram of load $P$ versus rotation $(-\theta)$ begins to descend at a finite slope $P_{c r} a$ [Fig. 3(a)]. On the other hand, the diagram of the load versus the associated displacement (i.e., the axial load-point displacement $u$ ) begins to descend with a vertical slope [Fig. 3(c)].

The postcritical behavior just illustrated is generally called asymmetric bifurcation, since the equilibrium path $P(\theta)$ or $P(u)$ at the critical point bifurcates in an asymmetric manner (symmetry would require a horizontal slope at $\left.P_{r r}\right)$. An important consequence is that the imperfect column has a maximum load $P_{\max }$ that is less than $P_{c r}$. The larger the imperfection, the smaller $P_{\max }$ is [Fig. 3(d)]. [Note that the American Concrete Institute (ACI) requires the columns to be designed for approximately $e>0.01 l$ even if the load is supposed to be centric.] Applied loads in buildings can often cause $e$ to be a large as $0.3 l$ or more.

The validity of Eq. 18 where $a$ is the initial slope, and especially the fact that $P_{\max }$ declines in proportion to $\sqrt{\alpha}(\alpha=$ imperfection), is not limited to this example. It represents the well-known half-power law of Koiter (1945), which applies generally to all asymmetric bifurcations. This law implies a rather severe sensitivity of the maximum load to the magnitude of imperfection, the severity being manifested by the fact that the curves in Figs. $3(c, d)$ start to descend with a vertical tangent (for imperfection-sensitive structures for which the bifurcation is symmetric, Koiter derived a 2/3-power law, $P_{\max }-P_{\mathrm{cr}} \sim \alpha^{2 / 3}$, which is less severe than $\left.\alpha^{1 / 2}\right)$.

The frame analyzed here was tested by Roorda (1965). Fig. 4 shows his test results for two different eccentricities $e$, which are above and below the value $e_{0}$ that offsets the geometrical imperfections of the model (adjustment of the theoretical curve by horizontal shift in Fig. $4(b)$ is necessary due to inevitable imperfections of the experiment). Using a perturbation technique, Roorda and Chilver (1970) analyzed this frame, which had previously been studied by means of an energy approach (Koiter 1967). They found the initial slope of the load-rotation curve to be $(d P / d \theta) / P_{c r}=0.381$ (at $\left.\theta=0\right)$,

2853

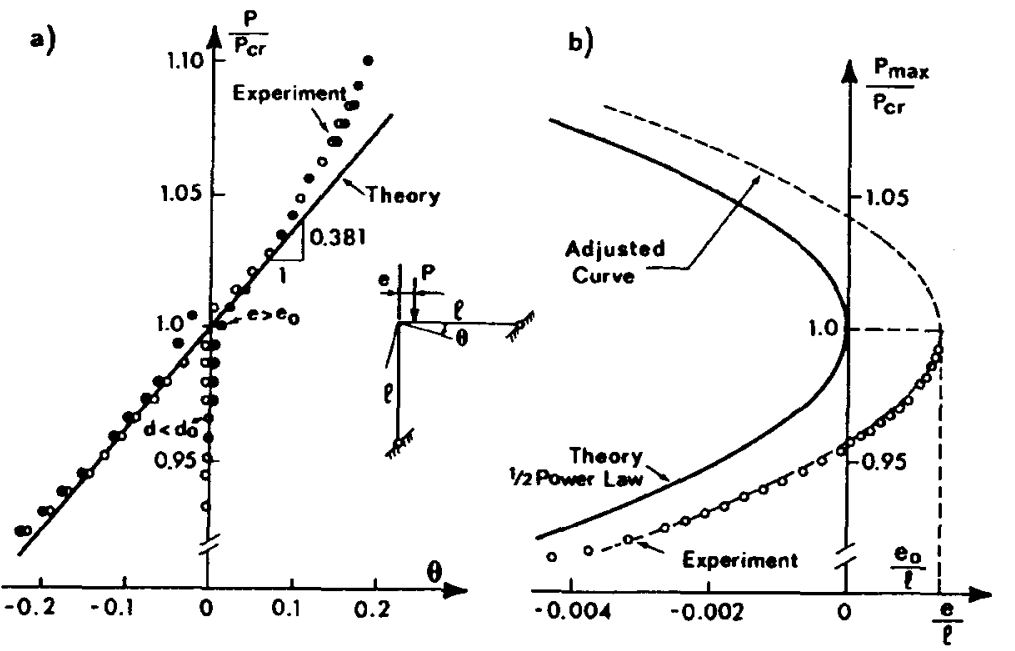

FG. 4. Experimental Results of Roorda $(1965 a, b)$

which agreed with Roorda's experimental results (see Fig. 4). The present calculation gives $a=0.379$, which might be more accurate, since the present method is more direct.

If for example $e=0.01 l$, then $\alpha=0.00871$, and Eq. 18 with $a=0.379$ yields $P_{\max }=0.885 P_{c r}$; i.e., $P$ drops by about $12 \%$ below $P_{c r}$. Calculation of this kind of drop in $P_{\max }$ has not been the practice in the design of frames.

\section{Generalizations and Implications}

To generalize the foregoing procedure to arbitrary frames one needs to consider second-order joint displacements due to lateral deflections, and the second-order changes of the stiffness coefficients due to second-order changes of the axial forces in members. The solution may be expected to lead in general to a system of quadratic equations.

Note that the power-series expansion of beam curvature, which reads $1 /$ $\rho=w^{\prime \prime}\left(1+w^{\prime 2}\right)^{-3 / 2}=w^{\prime \prime}\left[1-(3 / 2) w^{\prime 2}+(15 / 4) w^{\prime 4}-\ldots\right]$, lacks the second-order term $w^{\prime \prime} w^{\prime}$. The first term beyond the linear term $w^{\prime \prime}$ is the term $w^{\prime \prime} w^{\prime 2}$, which is of the third order, and so it affects in the joint equilibrium condition only terms of order $\theta^{3}$ but not of order $\theta^{2}$. It is for this reason that the second-order axial shortening can be calculated from the first-order deflection solution based on the linear curvature expression $1 / \rho \simeq w^{\prime \prime}$ and the linear stability functions $s$ and $c$. For the same reason, functions $s$ and $c$ (linear stiffness) are insufficient to determine initial postcritical behavior in symmetric bifurcations, for which third-order accuracy is required. Thus the symmetric bifurcation is harder to analyze than the asymmetric one. A higherorder accuracy is needed.

Since the bifurcation in columns is symmetric, the postbuckling solution for columns must involve at least a third-order approximation in $w$ (e.g., Thompson and Hunt 1973). Due to symmetry of the column buckling prob- 
a)

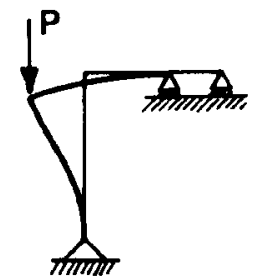

b)

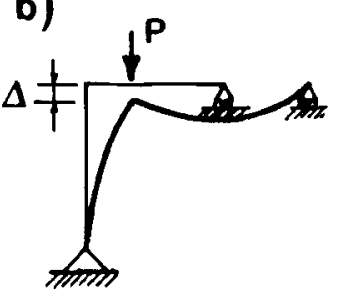

c)
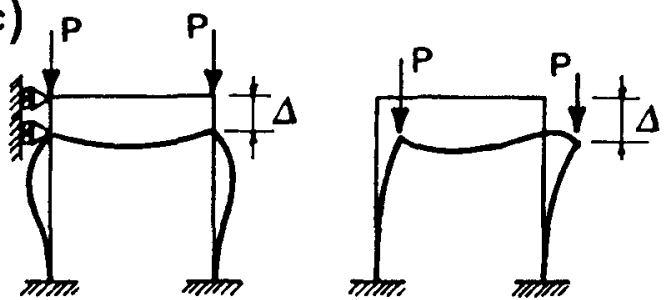

d)
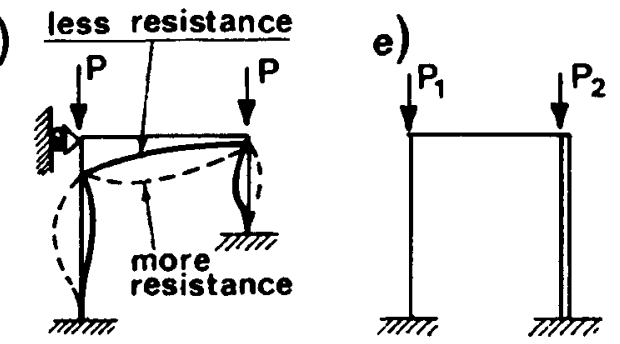

FIG. 5. (a,b) Asymmetrlc Frames; (c) Symmetric Frame; (d,e) Asymmetric Frames

lem, the second-order terms in $w$ cancel out. For the present L-frame, they do not cancel out, due to nonsymmetry; consequently the third-order terms in $w$ are not needed for the initial postcritical behavior. For this reason the present solution method (based on the $s$ and $c$ functions) could not be applied to postcritical behavior of a column.

In some frames, such as the symmetric portal frame in Fig. $5(\mathrm{c})$, the axial shortening meets with no resistance and the incremental shear force in the horizontal beam has no effect on the sum of vertical loads. Therefore, the bifurcation is symmetric, and neutral equilibrium exists at the critical state (for small deflections) just as it does for columns and also, obviously, for continuous beams. However, if portal frame columns have different lengths or different stiffnesses [Fig. $5(d, e)$ ], or different axial loads, their axial shortenings $u_{1}$ and $u_{2}$ are different and cannot be freely accommodated, and the incremental shear force in the horizontal beam affects the sum of the loads. Then the bifurcation is asymmetric. For frames the symmetric bifurcation is in fact an exception rather than a rule.

The redundancy of the frame per se is not the source of asymmetric bifurcation. For example, the two-bar frame in Fig. $5(a, b)$, which is statically determinate and is obtained from our previous frame by replacing the upper hinge support with a simple support, must also exhibit asymmetric bifurcation. Indeed, the buckling of the column to the left [Fig. $5(a)]$ in this frame also meets with less resistance than buckling to the right [Fig. $5(b)$ ]. The reason is that it produces less curvature in the horizontal beam, because the vertical column shortens in proportion to $\theta^{2}$.

Asymmetric bifurcation is also exhibited by a single simply supported column if the sliding plane of one support is misaligned with the beam axis. Since very small misalignments are inevitable, a small bifurcation asymmetry must always be present

\section{Conclusions}

1. The source of bifurcation asymmetry in nonsymmetric rectangular frames consists of: (1) The incremental shear forces transmitted to the columns from the adjacent beams as well as to the beams from the adjacent columns; and (2) the joint displacement caused by second-order axial shortenings of members due to their deflections.

2. A considerable simplification of the analysis is achieved by using the stiffness matrices that are known from the linear buckling analysis of frames and are based on the stability functions $s$ and $c$. The analysis necessitates calculation of certain second-order terms that involve derivatives of the stability functions with respect to the axial forces in members evaluated at the critical state of the perfect frame.

3. The present analysis agrees with Koiter's half-power law for the decrease of the maximum load at asymmetric bifurcation as a function of the imperfection, such as load eccentricity.

4. The example of the Koiter-Roorda L-frame shows that the present method of analysis closely agrees with the previous calculations of Koiter, and Roorda and Chilver, as well as with Roorda's experiments.

5. The reduction of the maximum load due to imperfections in frames exhib iting asymmetric bifurcation can be quite severe. In the present example, a load eccentricity of $1 \%$ of the column length causes a $12 \%$ drop in the maximum load, compared with the elastic critical load of a perfect frame.

6. Neglect of asymmetric bifurcation causes the safety factor in the frames exhibiting this type of behavior to be systematically less than in those that do not exhibit asymmetric bifurcation. Therefore, in the interest of uniform safety, the imperfection sensitivity due to asymmetric bifurcation ought to be taken into account in the design of frames.

\section{ACKNOWLEDGMENT}

The initial conception of the present formulation was made in connection with the work on NSF Grant ENG75-14848 to Northwestern University.

\section{Appendix. References}

Bažant, Z. P., and EINimeiri, M. (1973). "Large deflection spatial buckling of thinwalled beams and frames." J. Engrg. Mech. Div., ASCE, 99(6), 1259-1281. Dean, D. L., and Ugarte, C. P. (1968). "Field solutions for two-dimensional frameworks." Int. J. Mech. Sci., 10, 315-339. 
Horne, M. R., and Merchant, W. (1965). The stability of frames. Pergamon Press, London, U.K., 179.

Koiter, W. T. (1967). "Postbuckling analysis of simple two-bar frame, recent progress in applied mechanics." B. Broberg et al., eds., Folke Odquist Vol., Almquist and Wiksell, Göteborg, Sweden, 337.

Kounadis, A. N. (1985). "An efficient simplified approach for the nonlinear buckling analysis of frames." AIAA J., 23(8), 1254-1259.

Roorda, J. (1965a). "Stability of structures with small imperfections," thesis presented to the University of London, at London, U.K., in partial fulfillment of the requirements for the degree of Doctor of Philosophy

Roorda, J. (1965b). "Stability of structures with small imperfections." J. Engrg. Mech. Div., ASCE, 91(1),87.

Roorda, J., and Chilver, A. M. (1970). "Frame buckling: An illustration of the perturbation technique." Int. J. Non-Linear Mech., 5, 235-246. 